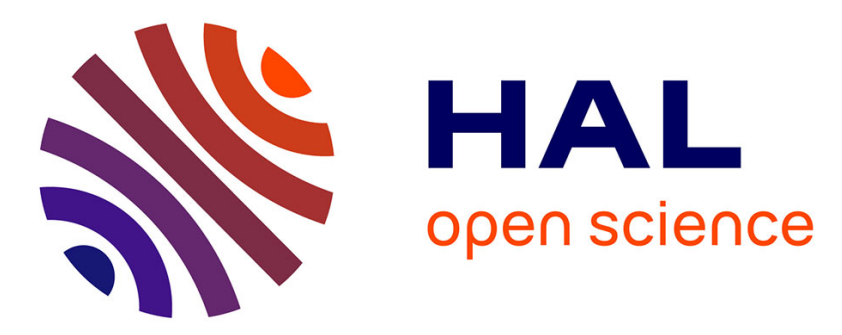

\title{
Impact of a Laser Pulse On HfO_2-based RRAM Cells Reliability and Integrity
}

\author{
A. Krakovinsky, Marc Bocquet, R. Wacquez, J. Coignus, D. Deleruyelle, C. \\ Djaou, G. Reimbold, Jean-Michel Portal
}

\section{- To cite this version:}

A. Krakovinsky, Marc Bocquet, R. Wacquez, J. Coignus, D. Deleruyelle, et al.. Impact of a Laser Pulse On HfO_2-based RRAM Cells Reliability and Integrity. 2016 INTERNATIONAL CONFERENCE ON MICROELECTRONIC TEST STRUCTURES (ICMTS), 2016, Unknown, Unknown Region. pp.152-156. hal-01435097

\section{HAL Id: hal-01435097 https://hal.science/hal-01435097}

Submitted on 28 Mar 2018

HAL is a multi-disciplinary open access archive for the deposit and dissemination of scientific research documents, whether they are published or not. The documents may come from teaching and research institutions in France or abroad, or from public or private research centers.
L'archive ouverte pluridisciplinaire HAL, est destinée au dépôt et à la diffusion de documents scientifiques de niveau recherche, publiés ou non, émanant des établissements d'enseignement et de recherche français ou étrangers, des laboratoires publics ou privés. 


\title{
Impact of a Laser Pulse On $\mathrm{HfO}_{2}$-based RRAM Cells Reliability and Integrity
}

\author{
A. Krakovinsky ${ }^{* \dagger \ddagger}$, M. Bocquet ${ }^{\dagger}$, R. Wacquez ${ }^{\ddagger}$, J. Coignus ${ }^{\ddagger}$, D. Deleruyelle ${ }^{\dagger}$, C. Djaou ${ }^{\dagger}$, \\ G. Reimbold ${ }^{\ddagger}$ and J-M. Portal ${ }^{\dagger}$
}

\begin{abstract}
Several NVM technologies have emerged during the last 10 years. These technologies offer solutions for the replacement of the Flash technology, which is facing downsizing limits [1]. Moreover these solutions propose lower switching energy and faster operations compared to the state of the art for Flash, and thus, are seen as an opportunity for the rise of the IoT market. But one of the main concerns regarding IoT is the protection of the data. Contrary to Flash, security of the data in emerging NVM is yet to be evaluated. In order to verify capability of the technology in terms of data integrity, we propose to investigate reliability and integrity of $\mathrm{HfO}_{2}$-based Resistive RAM (OxRRAM). This paper details the experimental protocol defined for laser-based attacks, shows that a laser pulse can affect the information stored in a single OxRRAM bit. The occurring phenomenon is then explained by mean of thermal and electrical simulations.
\end{abstract}

Index Terms-OxRRAM, Laser, Security, Integrity, $\mathrm{HfO}_{2}$, Simulation, Thermal Attacks, Optical Attacks.

\section{INTRODUCTION}

Innovative technologies are emerging as solutions for the future of Non Volatile Memories (NVM). One can cite Magnetoresistive Random Access Memories (MRAM), Phase Change RAM (PCRAM) or Resistive RAM (RRAM) as the main technologies of interest. $\mathrm{A} \mathrm{HfO}_{2^{-}}$ based RRAM solution is considered in this work. Due to its low cost of fabrication and its inherent lower switching energy, RRAM is of high interest for the IoT market.

In the following years, billions of smart objects will be interacting between each other. Power consumption is of course an essential issue. But if we consider the amount and nature of the processed data, the security aspect must not be neglected. In other words, emerging NVM must fulfill three essential criteria that are data integrity, data confidentiality and data accessibility. However, most studies still focus on the reliability of these technologies. Therefore none of them have been confronted to attacks such as UV lamp with masking [2] or focused laser [3] attacks that have been already shown as sucessful on Flash technology.

The first security criterion to be studied for NVM (especifically RRAM) is material integrity. This work proposes to evaluate the impact of external physical constraints on the material. Laser has been aleready proven able to disturb the behaviour of a circuit as well as old

*Corresponding Author - Phone Number : +33442616724 - Email address : alexis.krakovinsky@cea.fr

${ }^{\ddagger}$ CEA - DRT/DPACA, Laboratoire SAS, Centre de Microélectronique de Provence, Site Georges Charpak, 880 Avenue de Mimet, 13120 Gardanne, France And CEA LETI, Minatec Campus, 17 Avenue des Martyrs, 38054 Grenoble Cedex, France

${ }^{\dagger}$ IM2NP - UMR CNRS 7334, Aix-Marseille Université, Avenue Escadrille Normandie Niemen, Case 142, 13397 Marseille Cedex 20, France

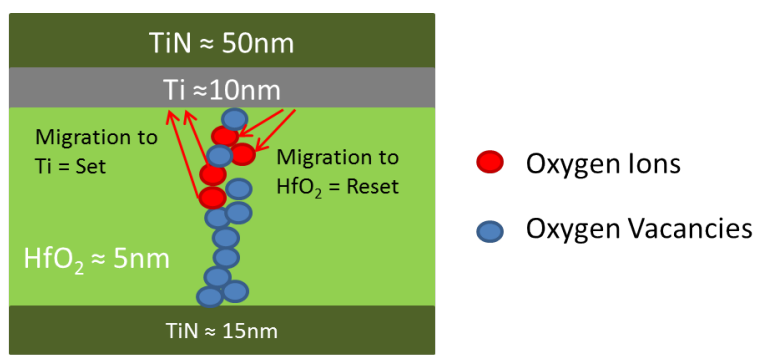

Fig. 1. Cell layer structure and ion migration description

Flash technology [4] through fault attacks. As a follow up of these works, it is interesting to attack RRAM in the same way.

Section 2 describes the experimental setup whose results are presented in section 3 and 4 . Section 5 is focused on the simulation of a laser pulse on a RRAM structure and of RRAM set operation.

\section{Experimental Setup}

\section{A. RRAM Principle and Characteristics Parameters}

A RRAM cell is made of two metal electrodes with a transition metal oxide (TMO) in-between. In our case (see Fig.1), these are made of a $5 \mathrm{~nm}$-thick $\mathrm{HfO}_{2}$ layer, located between a 10nm-thick Ti top electrode and a 10nm-thick TiN bottom electrode, as described in [5]. This technology relies on resistance switching, that is to say, in this case, migrating oxygen ions (as pictured in Fig. 1.) from the oxide to the electrode where a voltage is applied [6]. This process creates or dissolves a conductive filament $(\mathrm{CF})$ of oxygen vacancies in the oxide, which is tuning its resistance.

The way set and reset (i.e programming and erasing) operations are performed is presented on Fig. 2. $\mathrm{V}_{\text {stopreset }}$ and the set compliance current $\mathrm{I}_{c}$ are parameters which are set experimentally. For each experiment shown in this paper, $\mathrm{V}_{\text {stopreset }}=-1 \mathrm{~V}$ and $\mathrm{I}_{c}=1 \mathrm{~mA}$.

\section{B. Instrumentation and Experimental Protocol}

We use a laser bench with a Nd:YAG source. Three wavelenghths are available : $355 \mathrm{~nm}$ (Ultraviolet/UV), 533 $\mathrm{nm}$ (Green) and $1064 \mathrm{~nm}$ (Infrared/IR). It has a circular $50 \mu \mathrm{m}$-diameter spot size (as seen in Fig. 3.) that allows shooting the whole cell - whose size is $3 \mu \mathrm{m}$ up to a $400 \mu \mathrm{J}$ energy during a $10 \mathrm{~ns}$ pulse.

In the first place, to see the influence of laser pulses on the memory cells, their electrical characteristics were 


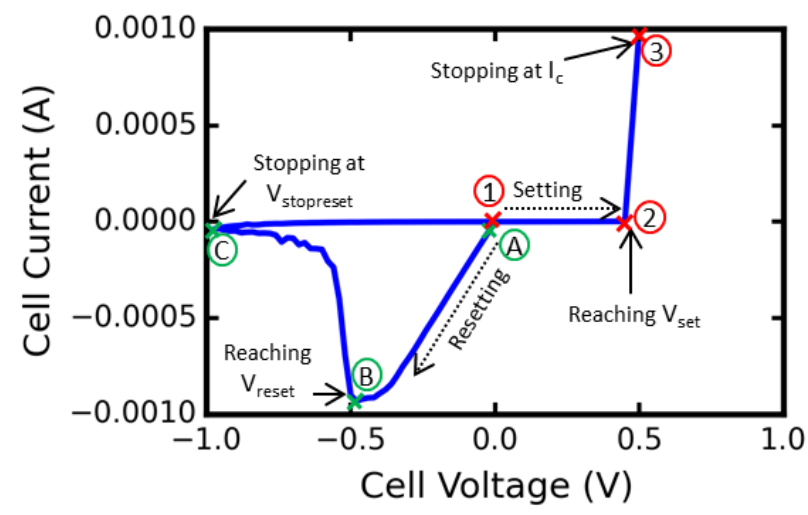

Fig. 2. Standard I-V characteristic of the $1 \mathrm{R}$ cells to be studied. For the set operation, a progressive voltage sweep is applied from ' 1 '. Then the cell switch to the LRS in '2' before its current reaches $\mathrm{I}_{c}$ in ' 3 '. For the reset operation, a voltage sweep is applied from 'A'. The cell switches from the LRS to the HRS in 'B'. The sweep is stopped at $\mathrm{V}_{\text {stopreset }}$ in ' $\mathrm{C}$ '

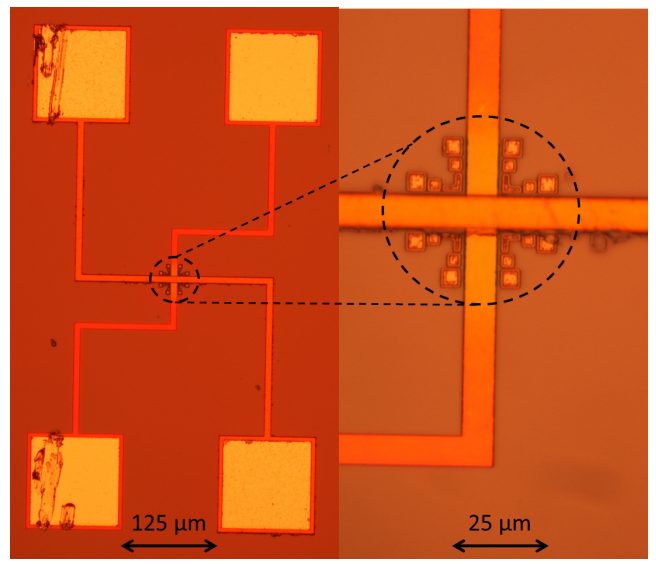

Fig. 3. Photographs of the whole structure including the aluminium pads (left) and the intersection of both metallization layers where the memristor is located (right). The laser spot size is represented by a circle on both pictures.

evaluated. These were conducted in quasi-static conditions (i.e without considerating temporal aspects) and consisted in 10 cycles of reset/set operations. The resistance value has been measured thanks to a read operation performed after each operation.

These preliminary measurements allow verifying that the cells have a regular behavor, in conformity with what has been published. These data will be then used as reference data for the following experiments (See Fig. 4.). The average set and reset voltages are respectively $\mathrm{V}_{\text {Set }}=0.6$ $\mathrm{V}$ and $\mathrm{V}_{\text {Reset }}=-0.54 \mathrm{~V}$. Moreover, the average LRS and HRS resistance values are about $\mathrm{R}_{L R \text { Smean }}=550 \Omega$ and $\mathrm{R}_{\text {HRSmean }}=35 \mathrm{k} \Omega$.

The experimental protocol of the next step is summed up in Fig.5. For the need of the experiment, $50 \%$ of all devices are left in HRS, and $50 \%$ in LRS. A single pulse in either UV or IR has been performed in order to check the potential impact of each wavelength. In the end, a
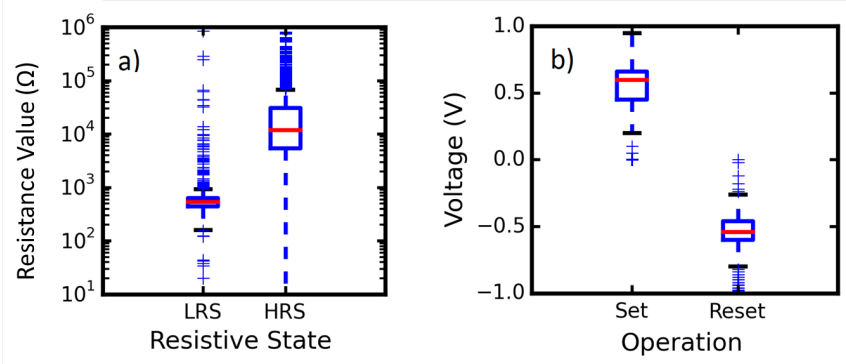

Fig. 4. Boxplot of 1500 LRS/HRS reference values (a) and Set/Reset voltages (b) obtained during preliminary characterisations of the studied cells. The box extends from the lower to upper quartile values of the data, with a line at the median.

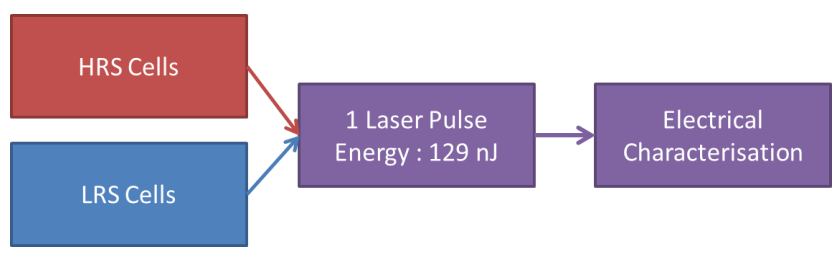

Fig. 5. Experimental Protocol Diagram

post-laser cycling will be made in order to compare the reference data to the new one.

\section{Influence on Resistance Values}

\section{A. Cells state variation and wavelength influence}

Regarding LRS cells after laser pulse, the first remarkable result is that the LRS remains unaffected. Fig. 6 shows that the cumulative distributions of the resistance values of the cells left initially in the LRS before and right after the pulse are similar. Moreover, both UV and IR data are overlaying.

However, contrary to LRS cells (as noticed on Fig. 7), a gap from about a decade in terms of resistance distribution can be seen between HRS cells before $\left(\mathrm{R}_{\text {meanbefore }}=\right.$ $34 \mathrm{k} \Omega)$ and after $\left(\mathrm{R}_{\text {mean }_{a} \text { fter }}=3400 \Omega\right)$ they were shot . Besides, half of these cells have switched to the LRS after the pulse.

Finally, as far as wavelength is concerned, UV and IR pulses have the same effect on HRS cells. Which means the laser impact on OxRRAM cells is independant from the wavelength used. Therefore, all the following data will not refer to the wavelength used during the experiment. The energy used will rather be taken into account as the most essential parameter.

\section{B. Laser Low Resistive State Analysis}

By confronting the resistance values of LRS cells to HRS cells that switched to the LRS cells after a laser pulse, it can be seen on Fig. 8 that the electrical LRS seems slightly different from the LRS obtained with laser. Indeed, the values of the switching cells $\left(\mathrm{R}_{L R S m e a n 1}=880 \Omega\right)$ are quite higher than those of the LRS cells $\left(\mathrm{R}_{L R S m e a n 2}=550 \Omega\right)$. 


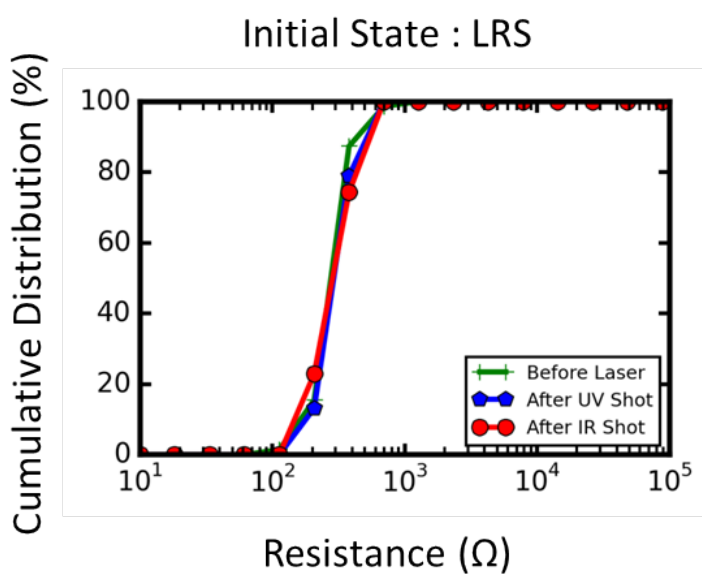

Fig. 6. Cumulative distributions of the resistance values of 84 LRS cells before and after a single laser pulse, sorted by wavelength used

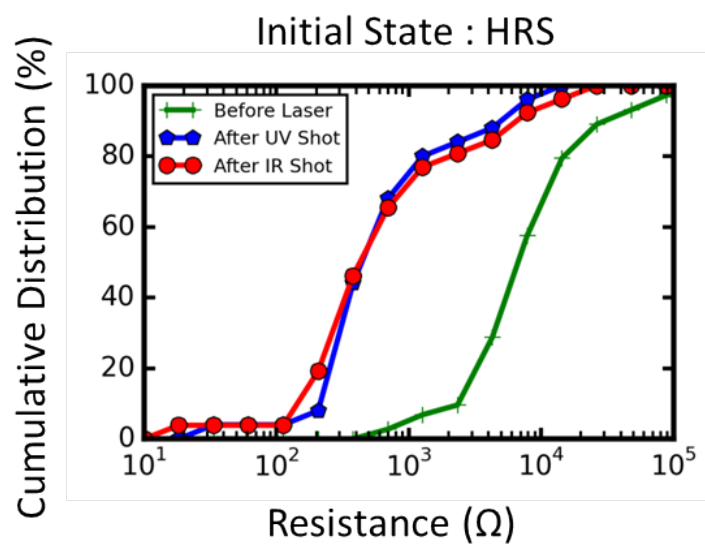

Fig. 7. Cumulative distributions of the resistance values of the 84 HRS cells before and after a single laser pulse, sorted by wavelength used

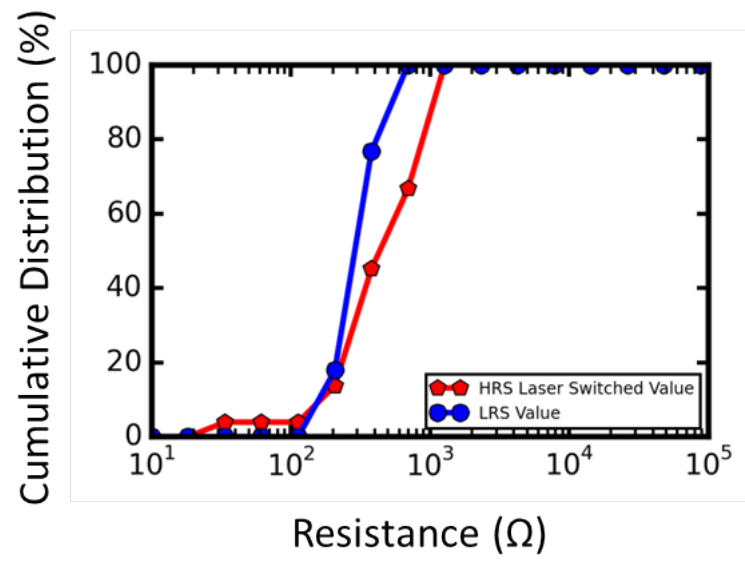

Fig. 8. Cumulative distribution of the resistance values of 40 cells that switched from the HRS to the LRS while being shot compared to the resistances values of cells set electrically to the LRS

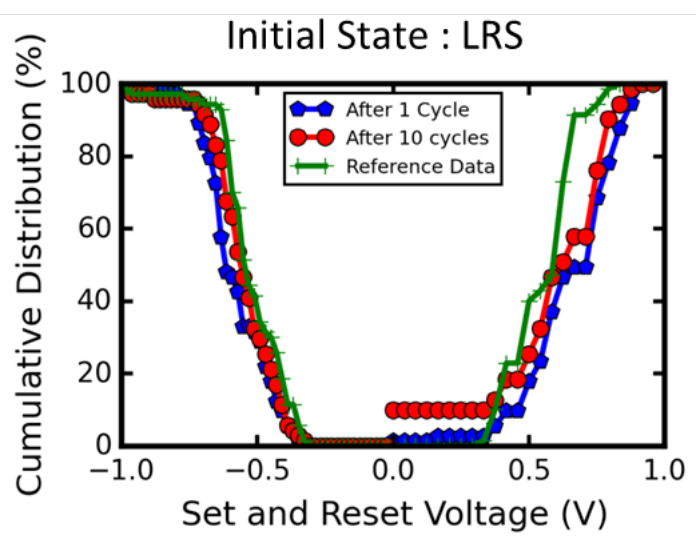

Fig. 9. Cumulative distribution of set and reset voltages of LRS cells after 1 or 10 Reset/Set cycles. These results were compared to reference data

In a nutshell, this analysis showed that a laser pulse can disturb the behaviour of only HRS cells. Nevertheless, the nature of the resistive state obtained after exposition is yet to be confirmed.

\section{Impact on Electrical Characteristics}

\section{A. Cells left in the LRS}

Even though the resistance values of LRS cells are showing the non-effectiveness of a laser beam on their state, $\mathrm{V}_{\text {Set }}$ and $\mathrm{V}_{\text {Reset }}$ measured after the attack may vary from those obtained before. But as expected, laser has not disturbed the cells since $\mathrm{V}_{\text {Set }}$ and $\mathrm{V}_{\text {Reset }}$ are matching the reference data (see Fig. 9).

\section{B. Cells left in the HRS}

Concerning the cells left in the HRS state, it has to be figured out whether or not cycling can be performed normally and if the laser set operation can be considered as equivalent to an electrical set operation. The cells that were submitted to a reset operation in a first place were hardly set to the HRS, since more than $80 \%$ of our devices first $\mathrm{V}_{\text {Reset }}$ are above the average voltage reset according to the reference data (as pictured in Fig. 10). This means laser set and electrical set are not equivalent, which is confirmed by the data coming from cells whose first post-laser operation was a set operation. Indeed, their first reset voltage is corresponding to the reference data.

Explaining the phenomena responsible for laser switching will require a model of the laser impact on the structure. As well as a model of an electrical set operation so we can compare them.

\section{Simulations}

There are two possibilities to explain a change in the oxide structure by the use of a laser beam. The first one is an optical effect provided by the photon injection and the second one is a temperature effect. Indeed, experiments have shown [7] that high temperature operations tend to 


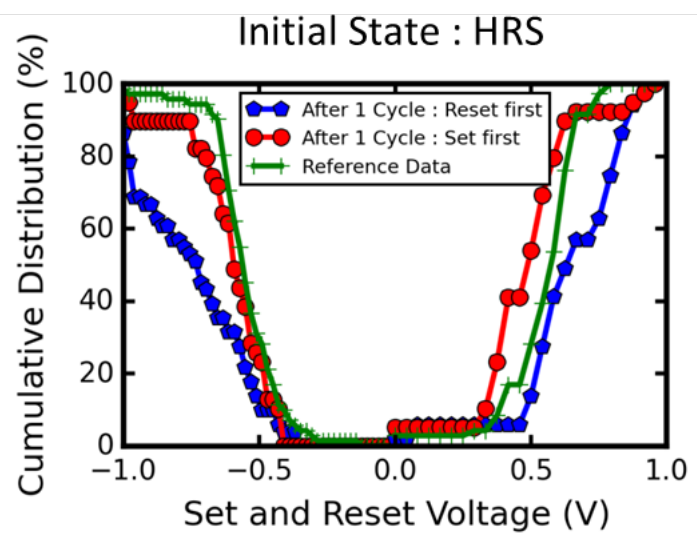

Fig. 10. Cumulative distribution of set and reset voltages of HRS cells after 1 Reset/Set cycle sorted by first operation performed . These results were compared to reference data

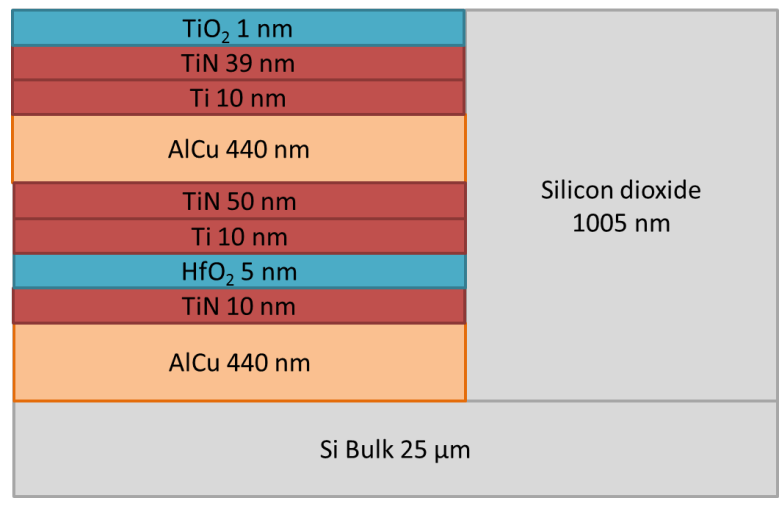

Fig. 11. Schematic view of the geometry used for thermal modeling

lower both $\mathrm{V}_{\text {Set }}$ and $\mathrm{V}_{\text {Reset }}$. Before any modeling work, we made an estimation of the optical penetration of the laser beam by calculating a simplified optical transmission coefficient for each layer of our structure, defined as follows :

$$
c=e^{\frac{-4 \times \pi \times k \times l}{\lambda}}
$$

Where $\mathrm{k}$ is the imaginary part of the refractive index of the material, 1 the material thickness and $\lambda$ the wavelength of the laser beam. For the metallization layer only (shown on Fig. 12), $\mathrm{c} \approx 1,7.10^{-15}$ for $\lambda=1064 \mathrm{~nm}$ and $\mathrm{c} \approx$ $4,3 \cdot 10^{-24}$ for $\lambda=355 \mathrm{~nm}$. In other words, photons are not able to go through the metallization layer and therefore reach the oxide. Which also means that instead of being caused by an optical effect, the laser switching shall be a consequence of temperature, the main focus of our modeling.

\section{A. Laser Modeling}

The geometry used for this model is presented on Fig. 11. For more accuracy, $1 \mathrm{~nm}$ of titanium dioxide has been considered on the top of our structure. The reason is since

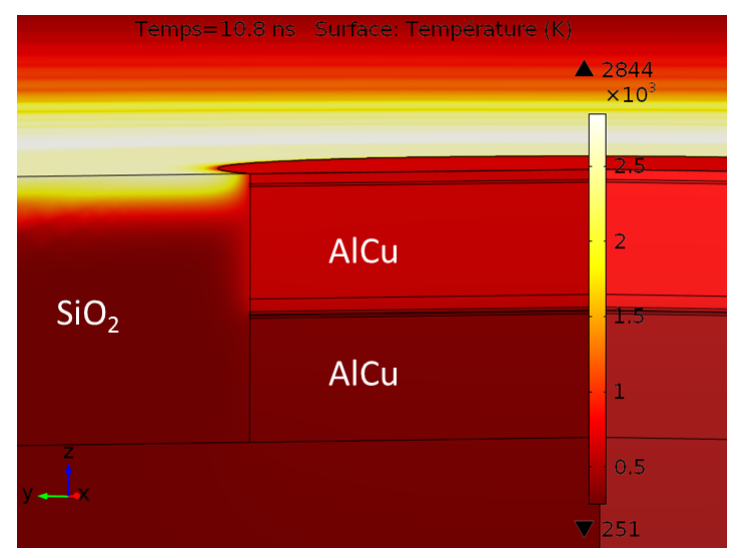

Fig. 12. Temperature repartition $0.8 \mathrm{~ns}$ after the $10 \mathrm{~ns}$ laser pulse on the whole structure

our measurements are performed in standard atmosphere, titanium nitride shall get oxidized which means we have a thin layer of oxide on the top of the structure.

Regarding the laser impact, the model was designed by using [8]. Therefore, the transmitted part of the laser energy to the surface of the structure and the heat emitted by each layer are respectively defined as follows :

$$
\begin{gathered}
I_{T}=I_{0}(1-R) g(x) \\
S(z)=\alpha \times I_{T L} \times e^{-\alpha z}
\end{gathered}
$$

Where $I_{0}$ is the laser surface power, $R$ the reflectivity of the surface material, $\mathrm{g}(\mathrm{x})$ the gaussian profile (whose standard deviation is a third of the laser spot radius) value for the $\mathrm{x}$ coordinate, $\alpha$ the optical penetration depth which equals $\frac{4 \pi k}{\lambda}$ (with $\mathrm{k}$ and $\lambda$ defined as in (1)), z the depth in the layer and $\mathrm{I}_{T L}$ the intensity transmitted to the layer top interface.

The simulation results show that the maximum temperature in the oxide is $610 \mathrm{~K}$ and that it is reached $0.8 \mathrm{~ns}$ after the end of the pulse (see also Fig. 12 and 13). This temperature is the result of the thermal diffusion coming from the titanium dioxide surface (whose maximum temperature is reached $0.8 \mathrm{~ns}$ before the end of the pulse). We might expect higher temperatures provided that the estimated silicon dioxide temperature is about $3000 \mathrm{~K}$ after the end of the pulse (beyond $\mathrm{SiO}_{2}$ melting point which is not taken into consideration in this model). However the duration needed for thermal diffusion from the $\mathrm{SiO}_{2}$ surface to the $\mathrm{HfO}_{2}$ layer is about $1 \mu$ s since silicon dioxide has a low thermal conductivity $\left(\mathrm{k}=1.4 \mathrm{~W} \cdot \mathrm{m}^{-1} \cdot \mathrm{K}^{-1}[10]\right)$ and the corresponding temperature much lower (about 500 $\mathrm{K})$.

The objective of the following electrical set modeling is to verify if the temperature reached during laser exposition is relevant for an electrical set operation. 


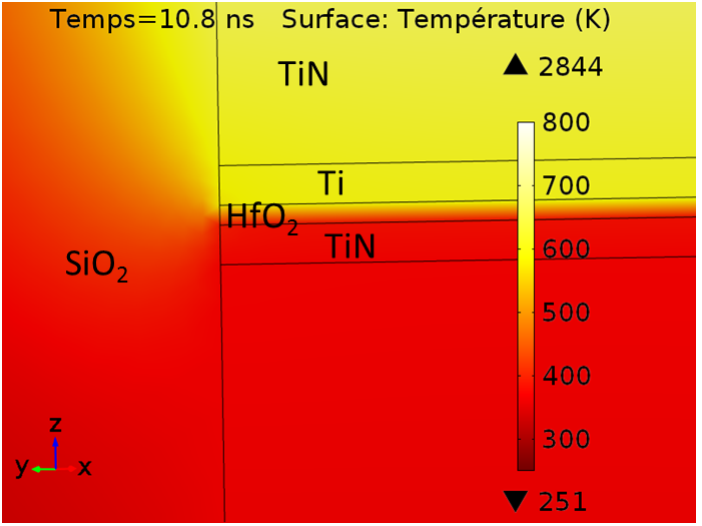

Fig. 13. Temperature repartition $0.8 \mathrm{~ns}$ after the laser pulse in the area around the hafnium dioxide layer

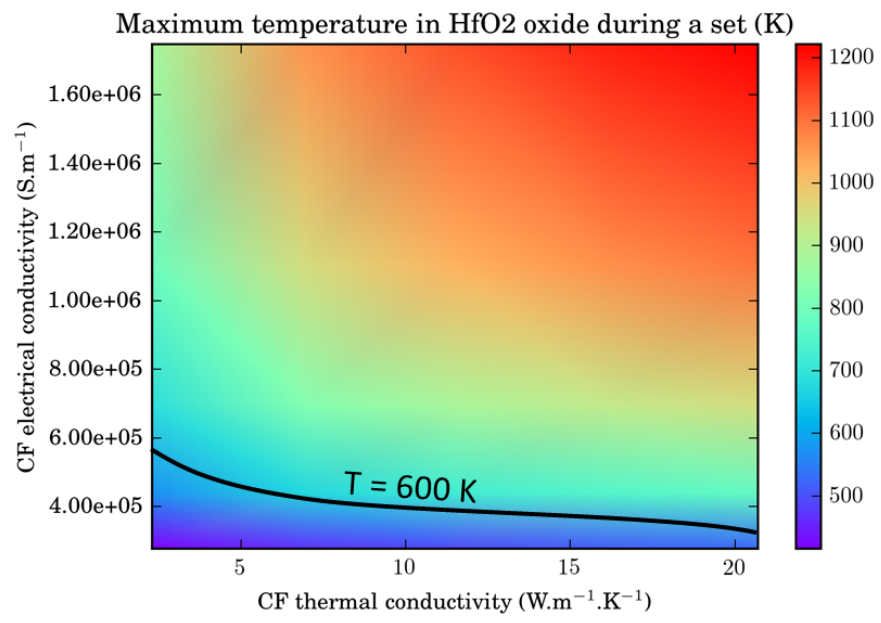

Fig. 14. Map of the temperature obtained in the $\mathrm{CF}$ when simulating an electrical set with a $10 \mu \mathrm{A}$ compliance current and a $0.5 \mathrm{~V}$ set voltage

\section{B. Electrical Set Simulation}

For the electrical set simulation, the model described in [9] has been applied to the cell structure. The main objective is to compare the temperature reached during an electrical set operation to the value obtained from thermal modeling. $\mathrm{V}_{\text {Set }}, \mathrm{I}_{c}$ as well as both thermal (k) and electrical $(\sigma)$ conductivities of the $\mathrm{CF}$ are the parameters of interest. By choosing the $\mathrm{CF}$ conductivities, we aim to get temperature according to its state after the set operation. In our case, the laser pulses were not able to really set the cells, which means the $\mathrm{CF}$ has a structure not far from $\mathrm{HfO}_{2}$, whose conductivities are lower than Hf.

We then simulated different conductivities values for each couple $\left.\left(\mathrm{V}_{\text {Set }}\right), \mathrm{I}_{c}\right)$ which gave us maps of the temperature reached in the $\mathrm{CF}$ in function of $\mathrm{k}$ and $\sigma$ at constant set voltage and compliance current. The values chosen for $\mathrm{V}_{\text {Set }}$ and $\mathrm{I}_{c}$ are respectively $0.5 \mathrm{~V} / 0.6 \mathrm{~V} / 1.2 \mathrm{~V}$ and 1 $\mu \mathrm{A} / 10 \mu \mathrm{A} / 0.1 \mathrm{~mA} / 1 \mathrm{~mA}$. Regarding the values of the conductivities, we decided to choose 10 values decreasing linearly (for k) and logarithmically (for $\sigma$ ) from the values given in [11] and [12] for Hf. The results presented on Fig. 14 were obtained for $\mathrm{V}_{\text {Set }}=0.5 \mathrm{~V}$ and $\mathrm{I}_{c}=10 \mu \mathrm{A}$.

The temperature calculated with laser modeling $(610 \mathrm{~K})$ is reached for $\mathrm{k} \approx 4 \mathrm{~W} \cdot \mathrm{m}^{-1} \cdot \mathrm{K}^{-1}$ and $\sigma \approx 510^{5} \mathrm{~S} . \mathrm{m}^{-1}$. These low conductivities mean that the set hasn't been totally completed since the CF structure is closer to hafnium dioxide than hafnium, explaining the experimental results obtained by laser pulse.

\section{CONCLUSION}

For the first time, RRAM cells have been disturbed by laser exposition, independently from the wavelength used. It is possible to perform a bitflip only from the HRS to the LRS. Simulations allowed us to explain that this phenomenon is due to the temperature brought by laser heating.

\section{REFERENCES}

[1] Takeuchi, K., Scaling challenges of NAND flash memory and hybrid memory system with storage class memory and NAND flash memory, Custom Integrated Circuits Conference (CICC), 2013 IEEE , vol., no., pp.1-6, 22-25 Sept. 2013

[2] Fournier, J.J.A.; Loubet-Moundi, P., Memory Address Scrambling Revealed Using Fault Attacks, Fault Diagnosis and Tolerance in Cryptography (FDTC), 2010 Workshop on , vol., no., pp.30-36, 21-21 Aug. 2010

[3] Skorobogatov, S., Local Heating Attacks on Flash Memory Devices Hardware-Oriented Security and Trust, 2009. HOST '09. IEEE International Workshop on, vol., no., pp.1-6, 27-27 July 2009

[4] Skorobogatov, S., Optical Fault Masking Attacks Fault Diagnosis and Tolerance in Cryptography (FDTC), 2010 Workshop on , vol., no., pp.23-29, 21-21 Aug. 2010

[5] Vianello et al. , Resistive Memories for Ultra-Low-Power embedded computing design, in Electron Devices Meeting (IEDM), 2014 IEEE International, vol., no., pp.6.3.1-6.3.4, 15-17 Dec. 2014

[6] Nardi et al. , Resistive Switching by Voltage-Driven Ion Migration in Bipolar RRAMPart I: Experimental Study, in Electron Devices, IEEE Transactions on , vol.59, no.9, pp.2461-2467, Sept. 2012

[7] Cabout et al. , Temperature impact (up to $200 \mathrm{C}$ ) on performance and reliability of HfO2-based RRAMs, Memory Workshop (IMW), 2013 5th IEEE International.

[8] David Sands (2011) Pulsed Laser Heating and Melting, Heat Transfer - Engineering applications, Prof. Vyacheslav Vikhrenko (Ed.), ISBN: 978-953-307-361-3

[9] Russo et al., Self-Accelerated Thermal Dissolution Model for Reset Programming in Unipolar Resistive-Switching Memory (RRAM) Devices, IEEE Transactions On Electron Devices, Vol. 56, N 2, February 2009

[10] Haynes, William M., ed. (2011), CRC Handbook of Chemistry and Physics (92nd ed.). Boca Raton, FL: CRC Press, ISBN 1439855110.

[11] David R. Lide (ed), CRC Handbook of Chemistry and Physics, 84th Edition. CRC Press. Boca Raton, Florida, 2003; Section 12, Properties of Solids; Thermal and Physical Properties of Pure Metals / Thermal Conductivity of Crystalline Dielectrics / Thermal Conductivity of Metals and Semiconductors as a Function of Temperature

[12] David R. Lide (ed), CRC Handbook of Chemistry and Physics, 84th Edition. CRC Press. Boca Raton, Florida, 2003; Section 12, Properties of Solids; Electrical Resistivity of Pure Metals

\section{Acknowledgements :}

This work was performed with the support of the CATRENE CA208 Mobitrust project. 